\title{
Spectral information retrieval from integrated broadband photodiode UV measurements
}

\author{
Luis Vázquez \\ Departamento de Matemática Aplicada. Facultad de Informática. Universidad Complutense de Madrid. 28040 Madrid. \\ Centro de Astrobiología, (CSIC-INTA). Carretera de Ajalvir km 4, 28850 Torrejón de Ardoz, Madrid, SPAIN \\ María-Paz Zorzano \\ Centro de Astrobiología, (CSIC-INTA). Carretera de Ajalvir km 4, 28850 Torrejón de Ardoz, Madrid, SPAIN
}

Salvador Jimenez

Departamento de Matemática Aplicada a las TT.II., E.T.S.I.T., Universidad Politécnica de Madrid, 28040-Madrid, SPAIN.

Compiled April 16, 2007

\begin{abstract}
We propose an algorithm to retrieve the global features of the spectral dependence of the ultraviolet (UV) irradiance from integrated, broadband UV measurements performed with a set of photodiodes with different UV filters. This fit, when applied to ground based measurements and compared to the incident Solar spectral irradiance on the top of the atmosphere, may be used to extract the spectral dependence of the UV opacity and the most relevant parameters characterizing the scattering with atmospheric aerosol (Angstrom exponent, etc) as well as the biological effective doses. In this way, using a set of photodiodes instead of a spectrophotometer, one may get spectral information within very low mass, package and weight constraints, which is particular useful for space missions. We consider its application for the rover-based exploration of the Martian ground which is subjected to daily and seasonal opacity variations. (c) 2007 Optical Society of America

OCIS codes: $280.1100,290.4020,040.7190,120.0120$
\end{abstract}

The ultraviolet (UV) radiative transfer problem in the Martian atmosphere is dominated by multiple scattering and absorption of photons with the micron-sized aerosols that are suspended in the thin atmosphere. Satellite and Martian-ground-based spectral measurements of ultraviolet (UV) radiation are important to retrieve accurate information of the atmospheric aerosols, in particular on aerosol size distribution, aerosol load, dust and cloud dynamics, and ozone content which in turn serves as a proxy for Mars atmospheric water vapor as well as to get a solid understanding of the UV radiation levels for chemical and biological implications. However any measuring device for outer Earth monitorization (either for satellites, landers or rover based measurements) is strongly limited by the mass, power, envelope and data allocation restrictions characteristic of the scientific payload of any space mission. With the rapid growth of optoelectronics technologies, photodiodes have been widely used in optical measuring systems, color measurement and analysis systems, etc. They have the advantage of being cheap, small in size and light (about $5 \mathrm{~g}$ weight and dimensions of less than $1 \mathrm{~cm}$ ). The photodiode package may include a filter that selects which photons wavelength of the incident irradiance are allowed to pass and get to the photodiode detector. The disadvantage of this system is that the photodiode gives an integrated measurement over a certain bandwidth, instead of the spectral information which is often desired but which would require the use of an spectrophotome- ter (between $200 \mathrm{~g}$ and $1 \mathrm{~kg}$ weight and dimensions of the order of tenths of $\mathrm{cm}$ ). This is a general problem in space mission instrumentation when weight, packaging, energy and data budget are severely constraint and adapted algorithms need to be implemented to extract scientifically relevant information from light and small sensors, such as thermopiles, instead of the spectral information of infrared spectrophotometers. ${ }^{1}$

In this letter we describe a general purpose algorithm to retrieve the global features of the spectral dependency of the UV irradiance in the $200-400 \mathrm{~nm}$ range, using the integrated signal of 5 different UV broadband photodiodes. Although this algorithm is of particular interest for the future ground-based exploration of Mars, it may be of general interest for other situations where low cost (in terms of mass, weight etc) spectral UV irradiance measurements are required. To date there are no in-situ measurements of the UV levels on the Martian ground surface. However the future Mars Science Laboratory (MSL) mission to Mars, NASA 2009, will include a light UV photodiode suite in the Rover Environmental Monitoring Station (REMS) sensor which will monitor the daily and seasonal variations of the UV ground level irradiance. The 5 photodiodes are here named UVC (208-280 nm), UV-B (280-320 nm) and UV-A (320$400 \mathrm{~nm})$, UV-D (245-290 nm) and UV-E (310-335 nm), and have similar wavelength ranges to those that shall be implemented in REMS. In REMS there will be an extra UV photodiode measuring the whole UV interval 
(200-400 nm) which may be used for redundancy checks.

To illustrate the way the algorithm works, we use a numerically obtained UV spectral irradiance, from a Radiative Transfer Code ${ }^{2}$ for a realistic operation scenario. We consider the UV radiative transfer problem for the Martian atmosphere, ${ }^{3}$ including the latest results on dust and cloud vertical distribution and Angstrom exponent $\mathrm{O}_{3}$ measurements from SPICAM on board of Mars Express satellite, and the measured visible opacities of the ground-based MER rovers as well as the UV ground albedo as seen by Mars Express aerosol UV scattering properties and the known solar incident irradiance at the top of the atmosphere (TOA), $I_{\text {solar }}(\lambda),{ }^{4}$ assuming, without loss of generality, equatorial latitudes and Mars at mean Mars-Sun distance (1.52 AU, for instance when it is close to the vernal equinox). Solving the Radiative Transfer Equation, with a fine layering multiple stream technique to accurately describe the influence of the aerosol vertical distribution one can estimate, among other things, the resulting UV direct $I_{d i r}$ and diffuse $I_{\text {diff }}$ spectral irradiance at the ground as would be measured on the Martian surface, at mid-latitudes or close to equatorial locations by future landers or rovers such as MSL. $^{3}$

The Martian atmosphere changes strongly with season and geographical location. As the $\mathrm{CO}_{2}$ polar caps sublime and condense the atmospheric pressure varies between 6 and 10 mbar. In addition this thin atmosphere is "loaded" with aerosols. The characteristic Martian micron-sized red dust is blown into the sky by winds (dust devils, dust plumes) and dust storms. In particular, after a dust storm the atmosphere is loaded with dust at planetary scales. The aerosol content is generally described by the opacity $\tau(\lambda)=-\ln \frac{I_{\text {dir }}(\lambda)}{I_{\text {solar }}(\lambda)}$ at a given wavelength. This expression describes the exponential reduction of the incident radiation at TOA propagating through the atmosphere until it reaches the ground. Here $I_{\text {solar }}(\lambda)$ is the incident radiation at TOA and $I_{\text {dir }}$ is the direct component of irradiance only, i.e. the solar radiation arriving to the ground in the direction of the Sun. Monitoring the daily and seasonal variations of this function one can describe the dust circulation and settling processes and estimate the absorption properties of dust. The spectral dependence of the aerosol optical depth can be characterized by the Angstrom's turbidity expression $\tau=\beta / \lambda^{\alpha}$, where $\alpha$ is the so-called Angstrom exponent and is related to the aerosol size and $\beta$ is the turbidity parameter and defines the aerosol optical depth at $1 \mu \mathrm{m} .^{5,6}$ Thus, knowing the spectral dependence of the opacity and extracting $\alpha$ one can estimate the average size of the dust in suspension. In Fig. 1 we show the incident solar irradiance on top $I_{\text {solar }}(\lambda)$, and the direct irradiance $I_{d i r}(\lambda)$ as would be measured in-situ at noon, when the atmospheric pressure is $6 \mathrm{mb}$, the $O_{3}$ level is 3 $\mu \mathrm{m}$-atm, in a "clear" dust scenario. ${ }^{3}$

However, as mentioned above, this spectrally resolved measurements will not be available. We will know instead the integral outputs from 5 photodiodes, in our

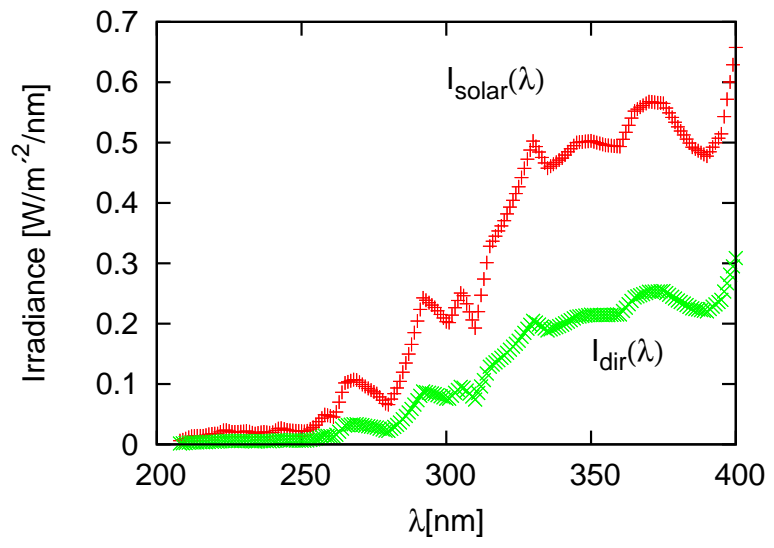

Fig. 1. Incident solar irradiance on top of the Martian atmosphere $I_{\text {solar }}(\lambda)$, and direct irradiance $I_{d i r}(\lambda)$ as would be measured in-situ at noon, see text for details.

particular test case: $C_{k}=\int_{\lambda_{k-1}}^{\lambda_{k}} I(\lambda) d \lambda, k=2,3,4$ with $\lambda_{1}=208 \mathrm{~nm}\left[1, \lambda_{2}=280 \mathrm{~nm}, \lambda_{3}=320\right.$ $\mathrm{nm}$ and $\lambda_{4}=400 \mathrm{~nm}$, together with $A=\int_{\mu_{1}}^{\mu_{2}} I(\lambda) d \lambda$, $B=\int_{\mu_{3}}^{\mu_{4}} I(\lambda) d \lambda$, and $\mu_{1}=245 \mathrm{~nm}, \mu_{1}=290 \mathrm{~nm}$, $\mu_{1}=310 \mathrm{~nm}, \mu_{1}=355 \mathrm{~nm} 2$. We want to recover a primitive function $f(\lambda)=\int_{\lambda_{1}}^{\lambda} I(x) d x$, satisfying the following conditions $f\left(\lambda_{1}\right)=0, f\left(\lambda_{k}\right)=C_{k}$ with $k=2,3,4$ and $f\left(\mu_{2}\right)-f\left(\mu_{1}\right)=A, f\left(\mu_{4}\right)-f\left(\mu_{3}\right)=B$. In this interpolation problem we will construct a polynomial $P(\lambda)$ of 5 th degree (since we have 6 conditions) that satisfies them and such that the function $I(\lambda)$ can be approximated by its derivative $Q(\lambda)=d P(\lambda) / d \lambda$ Let $P$ be $P(\lambda)=$ $a\left(\lambda-\lambda_{1}\right)^{5}+b\left(\lambda-\lambda_{1}\right)^{4}+c\left(\lambda-\lambda_{1}\right)^{3}+d\left(\lambda-\lambda_{1}\right)^{2}+e\left(\lambda-\lambda_{1}\right)$ and $Q(\lambda)=5 a\left(\lambda-\lambda_{1}\right)^{4}+4 b\left(\lambda-\lambda_{1}\right)^{3}+3 c\left(\lambda-\lambda_{1}\right)^{2}+$ $2 d\left(\lambda-\lambda_{1}\right)+e$. The unknown coefficients are obtained from:

$$
\left(\begin{array}{l}
a \\
b \\
c \\
d \\
e
\end{array}\right)=M^{-1}\left(\begin{array}{c}
C_{2} \\
C_{3} \\
C_{4} \\
A \\
B
\end{array}\right)
$$

were the matrix $\mathrm{M}$ is given by

$$
M=\left(\begin{array}{ccccc}
l^{5} & l^{4} & l^{3} & l^{2} & l \\
m^{5} & m^{4} & m^{3} & m^{2} & m \\
n^{5} & n^{4} & n^{3} & n^{2} & n \\
o^{5}-p^{5} & o^{4}-p^{4} & o^{3}-p^{3} & o^{2}-p^{2} & o-p \\
q^{5}-r^{5} & q^{4}-r^{4} & q^{3}-r^{3} & q^{2}-r^{2} & q-r
\end{array}\right)
$$

${ }^{1}$ The UV irradiance below $208 \mathrm{~nm}$ is strongly absorbed by the atmospheric $\mathrm{CO}_{2}$.

${ }^{2}$ In general, the sensor integral measurements will be an integral of the convoluted function $I(\lambda)=I^{*}(\lambda) f(\lambda)$, where $I^{*}(\lambda)$ is the incident radiation on top of the filter and $f(\lambda)$ is a previously callibrated and thus known (filter+detector) response function. As we we will later show, our interpolation algorithm allows us to retrieve the function $I(\lambda)$. Therefore, knowing the function $f(\lambda)$ we can easily recover the original $I^{*}(\lambda)$. Here for simplicity and without loss of generality we assume $f(\lambda)=1$ within the photodiode interval and 0 outside. 
with $l=\left(\lambda_{2}-\lambda_{1}\right), m=\left(\lambda_{3}-\lambda_{1}\right), n=\left(\lambda_{4}-\lambda_{1}\right)$, $o=\left(\mu_{2}-\lambda_{1}\right), p=\left(\mu_{1}-\lambda_{1}\right), q=\left(\mu_{4}-\lambda_{1}\right)$ and $r=\left(\mu_{3}-\lambda_{1}\right)$. As it will be later shown, and for the photodiode ranges chosen, this approximation works well on the higher range of the UV interval. We combine it with a rational interpolation which works well on the lower range of the UV interval, namely $S(\lambda)=P_{1}(\lambda) / P_{2}(\lambda)$ such that $I(\lambda)$ is approximated by $R(\lambda)=d S(\lambda) / d \lambda$. Having 6 conditions to be satisfied, we must impose that the degrees of the polynomial functions $P_{1}$ and $P_{2}$ should sum 4 (and none of the polynomials shall be equal to zero)..$^{7}$ If we ignore the rational functions that have a singularity in the range $\left[\lambda_{1}, \lambda_{4}\right]$ and those that become negative, the only option is that of $P_{1}$ being a polynomial of degree 1 and $P_{2}$ a polynomial of degree 3 . We proceed as before, imposing the conditions to be fulfilled and solving for the coefficients of the polynomials. In Fig. 2rabove we show the approximation to $I_{d i r}(\lambda)$ which is obtained from $R(\lambda)$ for $\lambda<300 \mathrm{~nm}$ and $Q(\lambda)$ for $\lambda>300 \mathrm{~nm}$. This interpolation deviates strongly on the UV-A range. We can use a linear combination of the two functions $a Q(\lambda)+b R(\lambda)$ such that $a+b=1$ and $a$ is taken such as to minimize the integral over the range $\left[\lambda_{1}, \lambda_{4}\right]$ of the square of the fit. This is shown in 2-below for $a=0.77$. The approximated function is indeed a soft interpolation of the direct spectral irradiance on the ground, where the characteristic peaks of the solar emission are softened and only the general trend is preserved.

This interpolated spectral irradiance is sufficiently good to obtain for instance the opacity and its spectral dependence, which as mentioned above, is one of the most relevant parameters to characterize the atmospheric dynamics. Next we apply the same procedure to obtain the interpolated functions of the incoming solar spectral irradiance $I_{\text {solar }}(\lambda)$, which is a priori known, and obtain the approximated functions $Q_{t o p}(\lambda)$ and $R_{t o p}(\lambda)$, which are "softened" versions of the incident solar irradiance. By doing this we can obtain the approximation of the opacity by either interpolating function $-\ln Q_{t o p}(\lambda) / Q(\lambda)$ or $-\ln R_{t o p}(\lambda) / R(\lambda)$ and compare with the "real" opacity $-\ln I_{\text {solar }}(\lambda) / I_{\text {dir }}(\lambda)$. As it is shown in Fig. 3 one approximation is better for one range and the other one for the other, but the result is coincident in the intermediate range and good enough to extract in addition to the spectral opacity other scientifically relevant parameters such as the Angstrom coefficient $\alpha$, which in this case turns out to be $\alpha \approx 1$. The difference in the $250 \mathrm{~nm}$ range is the signature of ozone absorption.

Finally, the total irradiance, incident on a normal surface is $I_{\text {total }}=I_{\text {dir }}+I_{\text {diff } f}$, where $I_{\text {diff }}$ is the irradiance that has been scattered either by Rayleigh scattering with atmospheric molecules or Mie scattering with atmospheric aerosols. Using the same Radiative Transfer model, we have estimated the total irradiance incident on a normal surface, as would be measured by the pho-
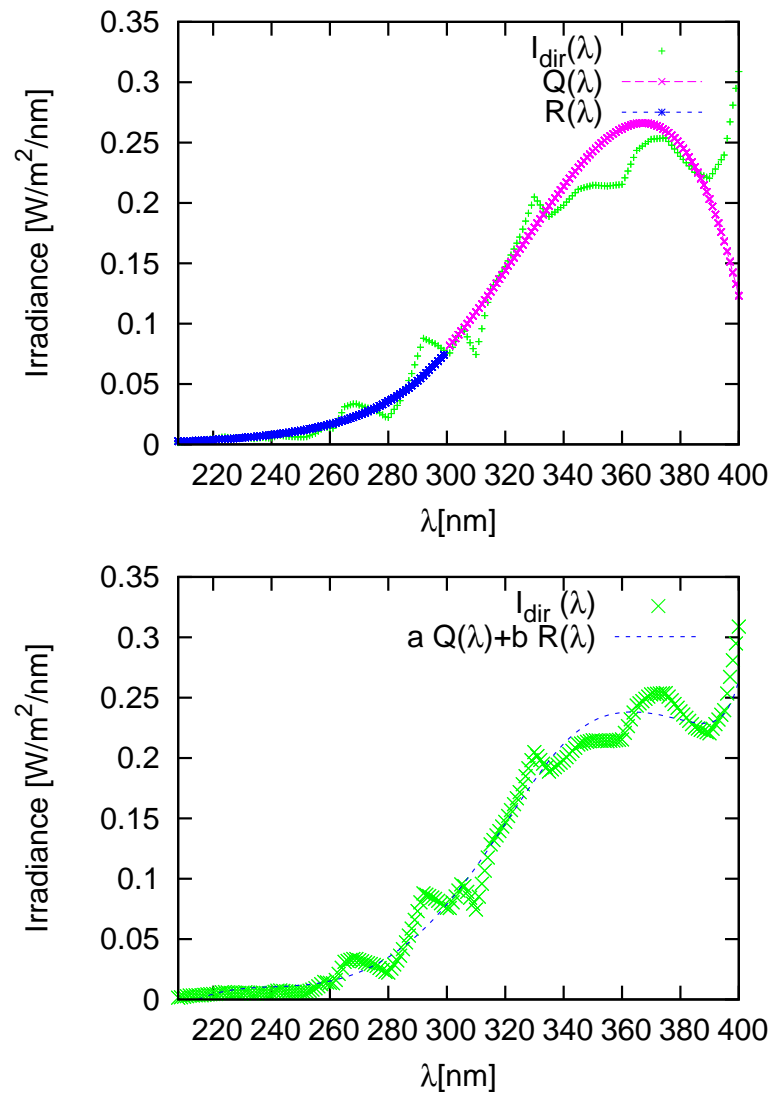

Fig. 2. Above: The interpolating polynomial $Q(\lambda)$ fits best the "real " signal $I_{d i r}(\lambda)$ in the interval $300-400 \mathrm{~nm}$, whereas the rational function $R(\lambda)$ fits best the "real" signal $I_{d i r}(\lambda)$ in the interval 200-300 nm. Below: The linear combination $a Q(\lambda)+b R(\lambda)$ with $a+b=1$ and $a=$ 0.77 is indeed a soft interpolation of the direct spectral irradiance on the ground.

todiodes 3. This global irradiance is, for instance, particularly relevant to estimate the biological impact that this UV irradiance will have on an specific target. The degree of absorption of radiation by important biological macromolecules, tissues or organisms, is wavelength dependent and as a result, the interfering effect of UV radiation is wavelength specific. The instantaneous (per second) biologically weighted dose $D=\int I_{\text {total }}(\lambda) B(\lambda) d \lambda$ is calculated from the convolution of the global irradiance $I_{\text {total }}(\lambda)$ incident on a surface with the Biological Action Spectrum $B(\lambda)$. In general, $B(\lambda)$ characterizes the sensitivity or biological response of an organism or biological structure (such as the one for DNA, or uracil, or specific proteins, or skin etc) to UV radiation. For instance the CIE action spectrum is a model for the susceptibility of the Caucasian skin to sunburn, ${ }^{8}$ which was adopted as a standard by the Commission Internationale de l'clairage (International Commission on Illumination): $B(\lambda)=1 \lambda<298 \mathrm{~nm}, B(\lambda)=10^{0.094(298-\lambda)}$

\footnotetext{
${ }^{3}$ The direct and diffuse component are distinguished by programming a direct Sun obscuration.
} 


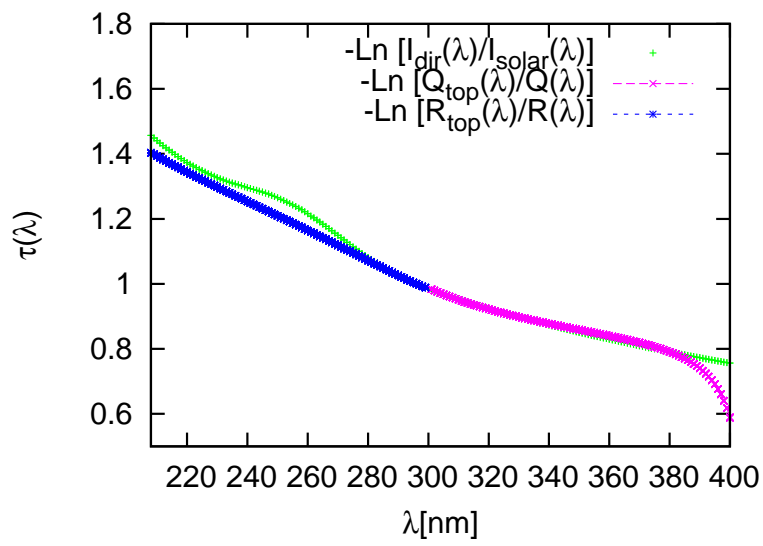

Fig. 3. Spectral dependence of the opacity. Comparison of the retrieved $\tau(\lambda)$ from the interpolated signal with the "real" one. The difference in the $250 \mathrm{~nm}$ range is the signature of ozone absorption.

$298 \mathrm{~nm}<\lambda<328 \mathrm{~nm}, B(\lambda)=10^{0.015(139-\lambda)} 328 \mathrm{~nm}<$ $\lambda<400 \mathrm{~nm}$. The multiplication of the irradiance with the CIE action spectrum gives the effective erythemal irradiance and its integral over the UV spectral range the widely used UV Index (UVI). In Fig. 4 we show the total irradiance, its interpolating function as retrieved from the 5 photodiodes and the multiplication of the interpolation by the CIE action spectrum, the area below the effective erythemal irradiance spectrum is the UVI. The value of the UVI would be of the order 2.76 for the interpolating function and 2.88 for the "real" total irradiance, which, for this particular scenario, corresponds to a maximum "safe" exposition-time of $167 /(U V I) \approx 60$ minutes on the surface of Mars.

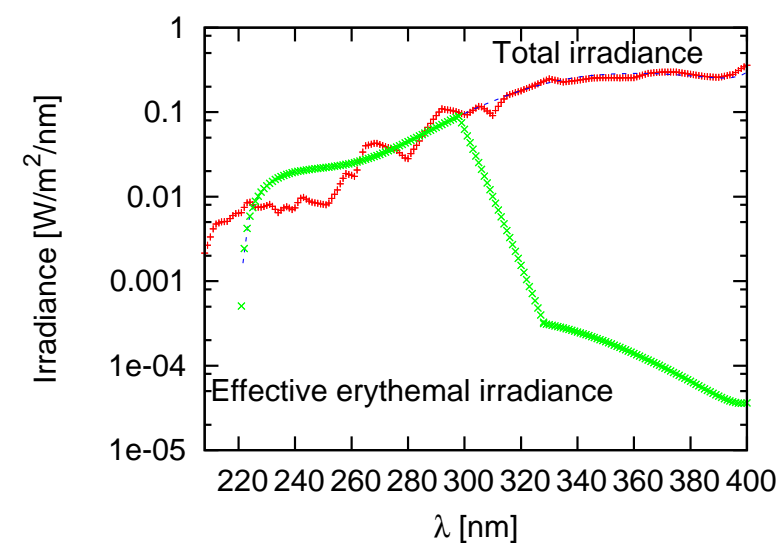

Fig. 4. Total (direct plus diffuse) UV irradiance on the Martian surface and retrieved interpolated function convoluted with the CIE action spectrum.

In this work we have shown how to obtain the spectral irradiance, including the general spectral features, from integrated broadband UV photodiode measurements. This interpolation is sufficiently good to describe aerosol opacity, aerosol Angstrom exponent (and thus aerosol size) and to evaluate biological relevant doses. Other features, such as ozone absorption, that require more spectral detail, need further refined algorithms.

M.-P. Z. and L. V. thank the support from the REMS project associated to the MSL-NASA mission to Mars and supported by the Centro de Astrobiología (CSIC/INTA). M.-P. Z. is supported by the Instituto Nacional de Técnica Aerospacial (INTA). S. J. has been partially supported by the Dirección General de Investigación of Spain, under Grant MTM2005-05573 (Spain).

\section{References}

1. Zorzano, M.-P and L. Vázquez, Remote temperature retrieval from heating or cooling targets, Optics Letters, pp. 1420-1422 (2006).

2. Zorzano, M.-P., Mancho, A.-M., Vazquez, L. 2005. Numerical integration of the discrete-ordinate radiative transfer equation in strongly non homogeneous media. Applied Mathematical and Computation, 164, 263-274.

3. Zorzano M.-P., Cordoba-Jabonero C. Influence of aerosol multiple scattering of ultraviolet radiation on Martian atmospheric sensing. Icarus in press (2007)

4. Thuillier G., Herse M., Labs D., Foujols T., Peetermans W., Gillotay D., Simon P. C. and Mandel H. The solar spectral irradiance from 200 to $2400 \mathrm{~nm}$ as measured by the SOSPEC spectrometer from the ATLAS and EURECA missions. 2003. Solar Physics, 214, 1-22.

5. Angstrom, A., 1929. On the atmospheric transmission of sun radiation and on dust in the air. Geographys. Annal. 2,156

6. Angstrom, A., 1961. Techniques of determining the turbidity of the atmosphere. Tellus 13, 214.

7. J. Stoer, R. Burlisch, Introdution to Numerical Analysis", second edition, ed. Springer-Verlag, New York 1993.

8. McKinlay and Diffey. A reference action spectrum for ultraviolet induced erythema in human skin, in Human Exposure to Ultraviolet Radiation: Risks and Regulations, pp. 83-87, Elsevier, Amsterdam, (1987) 